\title{
Anti-tumor Necrosis Factor Therapy for Crohn Disease: Friend or Foe to the Surgeon?
}

\author{
Hungdai Kim \\ Department of Surgery, Gastrointestinal Cancer Center, Kangbuk Samsung Hospital, Seoul, Korea
}

\section{See Article on Page 144-152}

Anti-tumor necrosis factor (anti-TNF) therapy is an effective therapeutic strategy for the induction and maintenance of remission in patients with Crohn disease (CD). Initially, anti-TNF therapy was employed as a last resort for patients with severe $\mathrm{CD}$, but it has now been recently accepted as a conventional option regardless of induction or maintenance of remission. Patients with $\mathrm{CD}$ are well known often to develop a number of severe complications, including bowel stricture/obstruction, perforation, intraabdominal abscess, and various forms of fistulas. Thus, most CD patients will have one or more abdominal operations in their lives. On that point, the role of anti-TNF therapy in the prevention of surgery or and its influence on postoperative outcome have drawn the attention of surgeons. Sakatani et al. [1] retrospectively analyzed the cumulative nonoperative rate of $104 \mathrm{CD}$ patients who had never had an abdominal operation until they had received their first surgery. They found that while infliximab (IFX) treatment extended the period from initiation of treatment to the first surgery, it did not reduce the risk of the first surgery.

Anti-TNF therapy also extends the duration from the previous to the next surgery. However, few data on the nonoperative rate of the next surgery in CD patient on anti-TNF therapy are available. Lee et al. [2] retrospectively reviewed the medical records of 148 CD patients who underwent abdominal surgery. They showed that use of anti-TNF therapy did not reduce the reoperation rate and that younger age at the first operation, penetrating behavior, and no azathioprine use were factors associated with reoperation. However, the lack of detailed information regarding anti-TNF

Correspondence to: Hungdai Kim, M.D.

Department of Surgery, Gastrointestinal Cancer Center, Kangbuk Samsung

Hospital, 29 Saemunan-ro, Jongno-gu, Seoul 31116, Korea

Tel: +82-2-2001-8541, Fax: +82-2-2001-2131

E-mail: hungdai.kim@samsung.com

(c) 2015 The Korean Society of Coloproctology

This is an open-access article distributed under the terms of the Creative Commons Attribution NonCommercial License (http://creativecommons.org/licenses/by-nc/3.0) which permits unrestricted noncommercial use, distribution, and reproduction in any medium, provided the original work is properly cited. therapy, such as the dose, schedule, or duration, may reduce the value of their conclusions.

In 2010, the European Crohn's and Colitis Organization made a statement on anti-TNF therapy that the combination of IFX with azathioprine was better than monotherapy for induction of remission and mucosal healing for up to 1 year in patients who are naive to both agents [3]. Also, patients who have a diminished or lower response to anti-TNF therapy may respond to dose adjustment of the same agent or switching to another agent. As the authors mentioned, anti-TNF therapy for patients with an early phase of CD is not covered by national insurance system of Korea. More work to extend coverage needs to be done by health care providers, as a result of which further prospective studies on $\mathrm{CD}$ patients with a proper anti-TNF therapy should become possible to confirm the significance of anti-TNF therapy in improving the rate of avoiding surgery.

Patients with bowel obstruction from stricturing-type CD warrant special consideration. Some questions have been raised that rapid luminal healing from IFX use leaves fibrosis and scarring of the bowel wall and leads to stenosis or obstruction. Lichtenstein et al. [4] analyzed data from the TREAT (the Crohn's Therapy, Resource, Evaluation, and Assessment Tool) Registry and the ACCENT I (A Crohn's Disease Clinical Trial Evaluating IFX in a New Long-Term Treatment Regimen) study. In the TREAT registry, stenosis or obstruction occurred at a significantly higher rate in patients treated with IFX compared with patients who received other treatments only. In multivariable analyses, however, IFX therapy was not associated with stenosis or obstruction development. In the ACCENT I study, no increase in stenosis or obstruction was reported in patients who received IFX maintenance therapy compared with those who received episodic therapy, despite a higher median cumulative IFX exposure. Although these data do not suggest that IFX therapy with or without rapid mucosal healing is associated with an increased risk of developing stenosis or obstruction, anti-TNF agents may likely induce stenosis or obstruction. Anti-TNF therapy should be used with caution in patients with stricturing-type CD.

An enterocutaneous fistula (ECF) develops in $20 \%$ to $40 \%$ of $\mathrm{CD}$ patients in their lifetimes. In the past, most $\mathrm{CD}$ patients with 
an ECF required surgical treatment. Amiot et al. [5] reporting on the long-term outcomes for CD patients with an ECF treated with anti-TNF therapy stated that complete ECF closure was achieved in 33\% of the patients after 3 months and long-term closure was achieved in $17 \%$ of the patients. Multiple ECF tracts and associated stenosis were independent factors for the 3-month complete ECF closure. Looking into their study, one other point claims our attention. That is, abdominal abscesses developed in $31 \%$ of the anti-TNF therapy group, and one patient died after surgery from abdominal sepsis and multiorgan failure.

Safety concerns have been raised with regard to the occurrence of serious infections in patients on anti-TNF therapy. Furthermore, increased septic perioperative outcomes of patients on IFX requiring surgery demand a more meticulous approach with caution. Appau et al. [6] evaluated preoperative IFX use and postoperative outcomes in CD patients. They concluded that IFX use within three months before surgery is associated with increased postoperative sepsis, abscess, and readmissions in CD patients. Thus, they recommended a diverting stoma for prevention of these complications. On the other hand, data from a systematic review of the same issue concluded that preoperative IFX use was not shown to be associated with higher rates of major postoperative complications in patients with CD undergoing intra-abdominal surgery. Minor complications, the need for a repeat operation and mortality within the first 30 days were also not affected by preoperative IFX therapy [7]. These results are somewhat reassuring in that patients who require surgery for management of their CD may be able to safely continue their IFX therapy during the preoperative period.

Putting studies together, anti-TNF therapy could extend the period from treatment to operation. However, it does not reduce the risk of surgery. In addition, preoperative anti-TNF therapy may not be as harmful as previously thought. Still, some loose ends need to be addressed before concluding that anti-TNF therapy does not have an effect on the surgery.

The feelings of patients on anti-TNF therapy should not be taken lightly!

\section{CONFLICT OF INTEREST}

No potential conflict of interest relevant to this article was reported.

\section{REFERENCES}

1. Sakatani A, Fujiya M, Ito T, Inaba Y, Ueno N, Kashima S, et al. Infliximab extends the duration until the first surgery in patients with Crohn's disease. Biomed Res Int 2013;2013:879491.

2. Lee SM, Han EC, Ryoo SB, Oh HK, Choe EK, Moon SH, et al. Long-term outcomes and risk factors for reoperation after surgical treatment for gastrointestinal Crohn disease according to antitumor necrosis factor- $\alpha$ antibody use: 35 years of experience at a single institute in Korea. Ann Coloproctol 2015;31:144-52.

3. D'Haens GR, Panaccione R, Higgins PD, Vermeire S, Gassull M, Chowers Y, et al. The London Position Statement of the World Congress of Gastroenterology on Biological Therapy for IBD with the European Crohn's and Colitis Organization: when to start, when to stop, which drug to choose, and how to predict response? Am J Gastroenterol 2011;106:199-212.

4. Lichtenstein GR, Olson A, Travers S, Diamond RH, Chen DM, Pritchard ML, et al. Factors associated with the development of intestinal strictures or obstructions in patients with Crohn's disease. Am J Gastroenterol 2006;101:1030-8.

5. Amiot A, Setakhr V, Seksik P, Allez M, Treton X, De Vos M, et al. Long-term outcome of enterocutaneous fistula in patients with Crohn's disease treated with anti-TNF therapy: a cohort study from the GETAID. Am J Gastroenterol 2014;109:1443-9.

6. Appau KA, Fazio VW, Shen B, Church JM, Lashner B, Remzi F, et al. Use of infliximab within 3 months of ileocolonic resection is associated with adverse postoperative outcomes in Crohn's patients. J Gastrointest Surg 2008;12:1738-44.

7. Rosenfeld G, Qian H, Bressler B. The risks of post-operative complications following pre-operative infliximab therapy for Crohn's disease in patients undergoing abdominal surgery: a systematic review and meta-analysis. J Crohns Colitis 2013;7:868-77. 